\title{
Focal High Signal on MR Scans of the Midbrain Caused by Enlarged Perivascular Spaces: MR-Pathologic Correlation
}

\author{
Allen D. Elster ${ }^{1}$ \\ Dan N. Richardson
}

Punctate and linear foci of abnormal signal were observed near the substantia nigra in the midbrains of $32(20 \%)$ of 157 patients undergoing high-resolution MR imaging of the brainstem. The lesions were most easily seen on long TR/long TE images, where they were of high signal intensity. Their location was consistently in the lower mesencephalon near the junction of the substantia nigra and cerebral peduncle. Unilateral lesions were observed in 18 cases, while bilateral lesions were noted in 14.

A review of anatomic specimens revealed the constant presence of penetrating branches of the collicular or accessory collicular arteries in this location. Enlarged perivascular spaces around these vessels were frequently seen in the specimens and probably account for punctate and linear foci observed on high-resolution MR images of the midbrain.

\section{AJNR 11: 1119-1122, November/December 1990; AJR 156 January 1991}

Punctate foci of high signal on T2-weighted images of the brain have long excited curiosity and interest. Initially dubbed "UBOs," or "unidentified bright objects," the anatomic and pathologic correlates of these lesions are gradually being explained. Known causes of these lesions include enlarged perivascular spaces, atrophic perivascular demyelination, white matter infarctions, capillary telangiectasias, congenital brain cysts, ventricular diverticula, ependymitis granularis, and isomorphic gliosis [1-9]. When no particular causative factor is identified, the descriptive term "leuko-araiosis" has been applied to these lesions [10].

Although these lesions were initially observed supratentorially, several investigators have noted that similar-appearing lesions may also be found in the brainstem $[1,6]$. These brainstem lesions are relatively common in elderly patients, are located predominantly in the pons, and frequently possess ill-defined margins. Recently, we began to observe a different type of brainstem lesion in several young patients undergoing high-resolution MR imaging of the posterior fossa. These lesions were punctate and linear foci typically located near the substantia nigra and cerebral peduncle in the lower mesencephalon (Fig. 1). Because of their consistent location and shape, we speculated that these midbrain lesions represented anatomic structures rather than being merely nonspecific white matter abnormalities. We therefore set out to investigate systematically the frequency of these lesions and to establish their true identity.

Received May 3, 1990; revision requested June 4, 1990; revision received June 18, 1990; accepted July 2, 1990.

1 Both authors: Department of Radiology, Bowman Gray School of Medicine, Wake Forest University, $300 \mathrm{~S}$. Hawthorne Rd., Winston-Salem, NC 27103. Address reprint requests to A. D. Elster.

0195-6108/90/1106-1119

(๑) American Society of Neuroradiology

\section{Materials and Methods}

During an 18-month period, 157 patients were referred to us for high-resolution MR studies of the cerebellopontine angle and temporal bone. The patients ranged in age from 28 to 82 years old (median, 45 years). Approximately $90 \%$ (141/157) of these patients were referred from our otolaryngology service for hearing loss or vertigo, with the principal clinical injunction to "rule out acoustic neurinoma." The remaining patients were referred for a variety of lesions involving the temporal bone, including postoperative complications, infections, and neoplasms. 

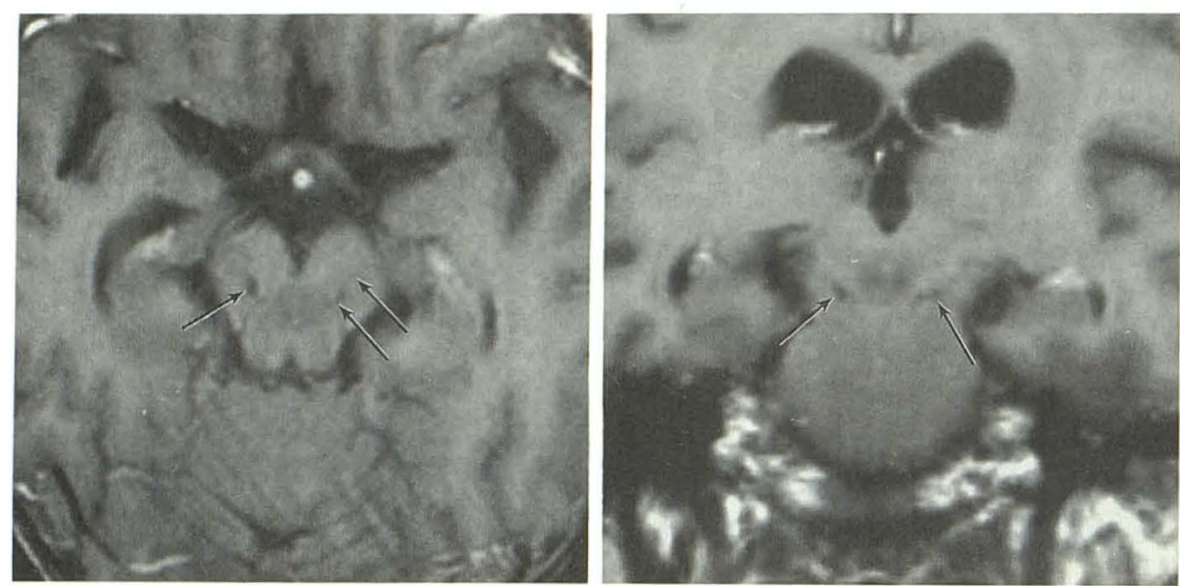

A

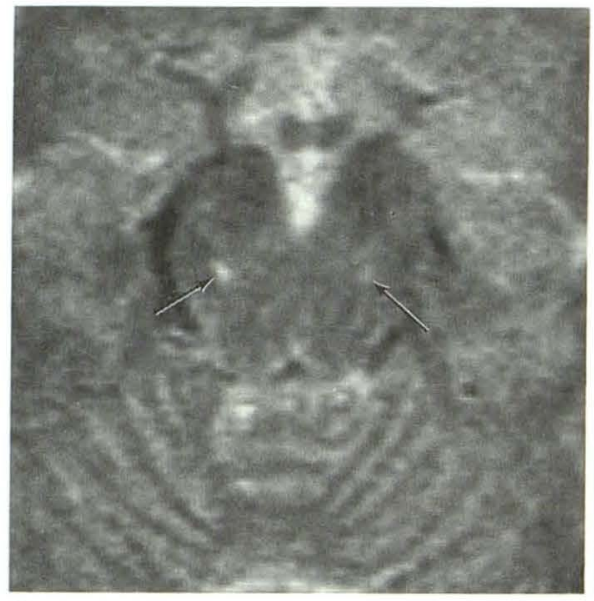

C
B

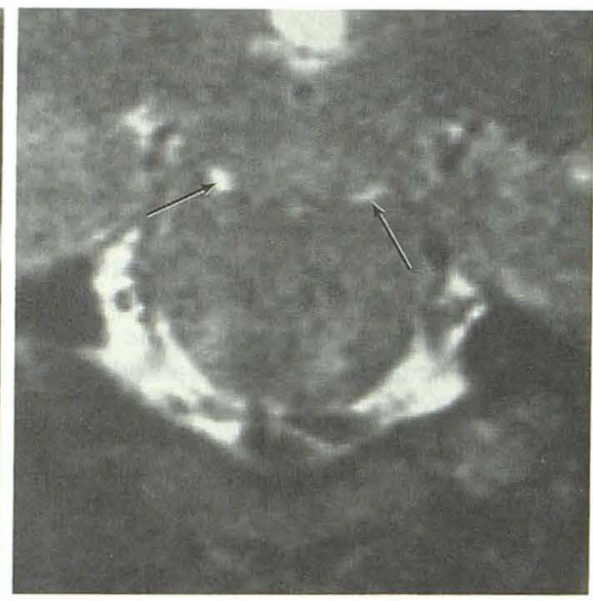

D

Fig. 1.-Punctate and linear foci (arrows in A$D)$ of abnormal signal seen in midbrain.

A, Axial T1-weighted image $(600 / 20 / 2)$. Lesion on left has a more linear configuration.

$B$, Coronal T1-weighted image $(600 / 20 / 2)$.

$C$ and $D$, Axial and coronal T2-weighted images $(2000 / 120 / 2)$.

No patient in this series had symptoms or signs referable to the midbrain, as determined by review of a three-page medical questionnaire completed by each patient prior to imaging.

All scans were acquired at $1.5 \mathrm{~T}$ (Picker Vista MR, Highland Heights, $\mathrm{OH}$ ). The scans analyzed were from a triple-echo protocol with parameters of $2000 / 40,80,120 / 2$ (TR/TEs/excitations). Slices were $3.5-\mathrm{mm}$ thick with a $0.5-\mathrm{mm}$ gap, and the images analyzed were exclusively from the coronal plane. Other scan parameters included a field of view of $20 \mathrm{~cm}$ and an image acquisition matrix of $192 \times$ 256. Axial T2- and T1-weighted images were inconsistently obtained through the upper midbrain, so these were not used in the statistical tabulation. Where available and appropriate, however, images from these sequences were retained for purposes of illustration. Similarly, since all patients were referred for suspected disease involving primarily the cerebellopontine angles or temporal bone, complete images of the rest of the brain were obtained only occasionally in fewer than 15 patients.

The MR scans were retrospectively reviewed by the authors to determine the frequency of punctate and linear foci seen in the lateral and inferior mesencephalon as illustrated in Figure 1. High-signal foci were scored by consensus of the readers as either present or absent in each case. If present, the lesions were further scored as being present unilaterally or bilaterally.

Following the retrospective analysis of the MR scans, we reviewed serial midbrain sections from 25 cadaver brains. These specimens were selected at random from a medical school anatomy laboratory collection, the ages and clinical status of the donors being unknown. All brains were fixed in formalin, then embedded in paraffin, sectioned at $40 \mu \mathrm{m}$, and stained with $\mathrm{H}$ and $\mathrm{E}$. The sections were viewed with low-power light microscopy using magnification of up to $\times 200$. Reference to a major brainstem anatomy text was made in order to assign specific identities to the structures visualized [11].

\section{Results}

Punctate and linear foci of high signal on T2-weighted MR images were identified in the midbrains of 32 (20.4\%) of 157 patients. In 18 cases the lesions were unilateral and in 14 cases they were bilateral. The lesions were consistently located in the lower mesencephalon near the substantia nigra, cerebral peduncle, and decussation of the brachium conjunctivum. Representative examples are shown in Figure 1.

The lesions seen were approximately $1-1.5-\mathrm{mm}$ in greatest dimension and were frequently more ovoid or linear than round. They were best seen on the TE 120 images, and (when performed) also on the T1-weighted images. On all pulse sequences used they maintained isointensity with CSF.

Since our study was not prospectively designed and since complete images of the rest of the brain were not routinely obtained, we did not attempt to correlate the presence of these midbrain lesions with supratentorial white matter lesions 
or enlarged perivascular spaces elsewhere in the brain. We found no significant differences in the ages of the patients with midbrain lesions (28-79 years) and those without such lesions (29-82 years). Since nearly all patients in both groups were referred for suspicion of acoustic neurinoma, there were likewise no significant differences between the two groups with regard to clinical symptomatology. Enlarged perivascular spaces near the anterior commissure were seen in association with the midbrain lesions in several patients who had complete brain studies; conversely, several patients had midbrain lesions as an isolated finding.

Review of the anatomic specimens revealed the constant (25/25 cases) presence of small, penetrating vessels in the inferolateral midbrain (Fig. 2). The vessels seen were identified as branches of the collicular and accessary collicular arteries, which are themselves branches of the posterior cerebral artery. In six of these specimens, prominent perivascular spaces measuring larger than $1 \mathrm{~mm}$ in greatest dimension were observed. These perivascular spaces were of the appropriate size, location, and orientation such that they could reasonably account for the punctate and linear foci of high signal seen on MR images of the midbrain.

\section{Discussion}

Over the last 2 years, several investigators have noted that enlarged perivascular spaces are responsible for producing punctate lesions on MR scans in the brains of otherwise healthy individuals [1, 2, 7-9]. These enlarged perivascular (Virchow-Robin) spaces are commonly seen in two locations: along the anterior commissure into the lower basal ganglia and at the brain vertex. The MR signal characteristics of these perivascular spaces are such that they maintain isointensity with CSF on all pulse sequences used. The midbrain lesions we observed have MR signal characteristics entirely consistent with that reported for perivascular spaces elsewhere in the brain.

Review of serial anatomic specimens and the anatomic literature reveals that the enlarged perivascular spaces in the midbrain we have observed most likely surround penetrating branches of the collicular and accessory collicular arteries (Fig. 3). The crus cerebri and anterolateral midbrain are supplied primarily by rami of these arteries. The collicular artery is a small but constant vessel that arises from the posterior cerebral artery in the interpeduncular fossa [11]. Here the artery lies in close relation to radicles of the oculomotor nerve.

Exiting the interpeduncular fossa the collicular artery first traverses the anterior surface of the crus cerebri, closely following the pontomesencephalic sulcus. Upon reaching the lateral margins of the crus, the collicular artery ascends aci uss its lateral side reaching the lateral mesencephalic sulcus. Thereafter, the artery inclines sharply posteriorly to reach the brachium of the inferior colliculus. Here it divides into its two terminal branches that supply the superior and inferior colliculi, respectively.

In most specimens an accessory collicular artery may also be found. This vessel branches from the proximal segment of the main collicular artery, following it closely upon its upper side as far as the lateral mesencephalic sulcus. Both the main and accessory collicular arteries give rise to numerous penetrating branches that supply the cerebral peduncle, substantia nigra, and lateral portion of the red nucleus. These arteries have a steeply oblique course through the brainstem, accounting for their segmental visualization on serial MR images.

Each penetrating mesencephalic artery is often closely accompanied by a satellite vein. Frequently, several veins and arteries are seen grouped into a small pedicle for some distance after penetrating the lateral margin of the crus cerebri. The penetrating veins drain outward to the lateral mesencephalic vein. This vessel in turn links the basal vein to the superior petrosal vein. We were unable to observe any venous structures of size comparable to the perivascular spaces surrounding the collicular arteries in any of our anatomic specimens. It remains possible, however, that in some cases signal from slowly flowing blood in the satellite veins described
Fig. 2.-Anatomic specimens ( $\mathrm{H}$ and $\mathrm{E}$ stain) of midbrain $(m)$ and pons $(p)$.

$A$, Coronal section $(\times 5)$. Enlarged perivascular spaces surrounding collicular artery are seen on left (double arrows). A normally sized perivascular space is noted on right (single arrow).

$B$, Higher power view of $A(\times 50)$. Branches of collicular artery (a) are seen surrounded by enlarged Virchow-Robin space. The perivascular space is lined by pia (arrowheads). Note pigmented cells of substantia nigra nearby (arrows).

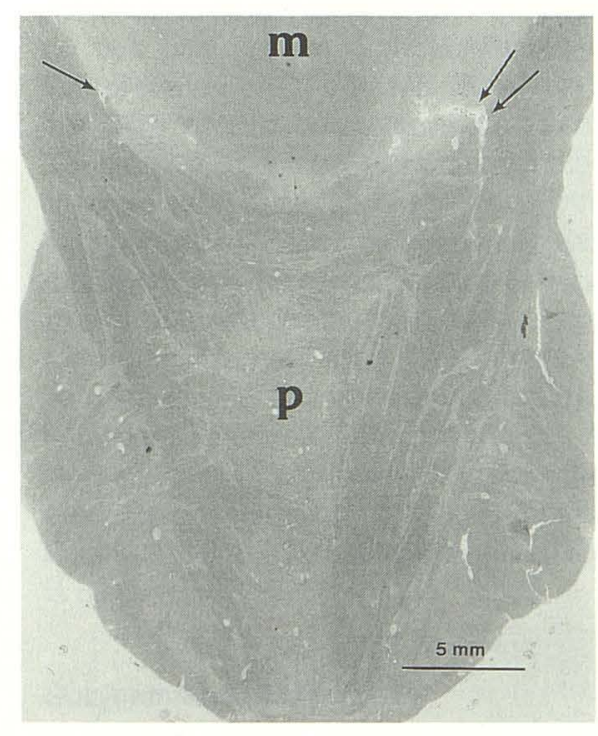

A

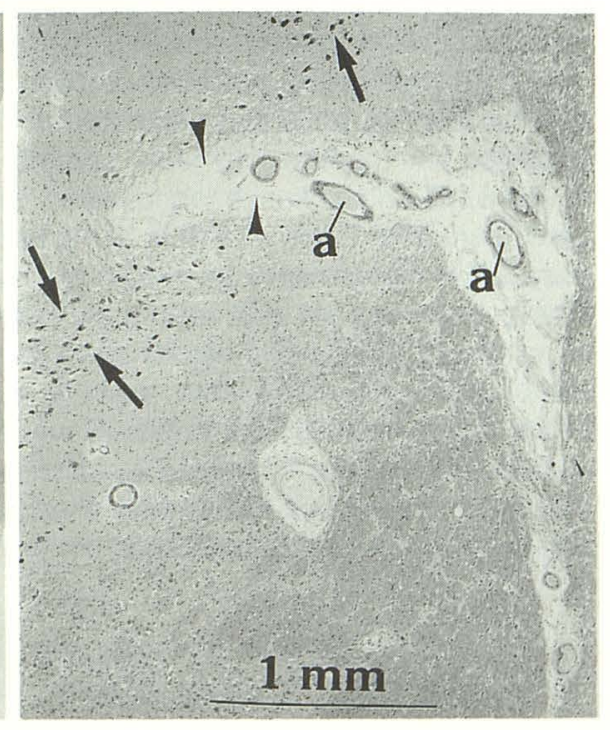

B 


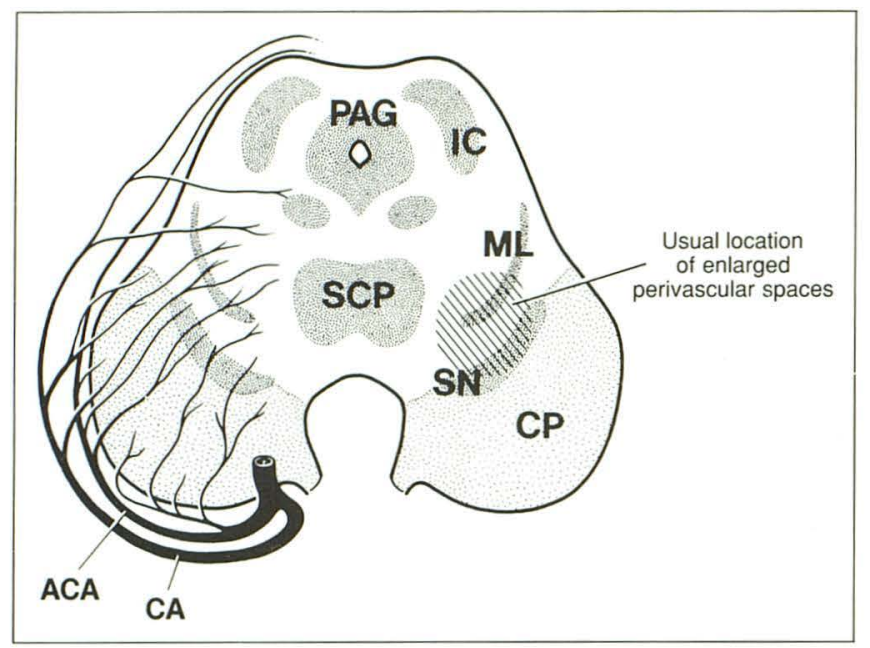

Fig. 3.-Diagram of blood supply to lateral midbrain. Penetrating branches of collicular artery (CA) and accessory collicular arteries (ACA) routinely supply the region where enlarged perivascular spaces are found. $\mathrm{CP}=$ cerebral peduncle, $\mathrm{SN}=$ substantia nigra, $\mathrm{ML}=$ medial lemniscus, $\mathrm{SCP}=$ decussation of superior cerebellar peduncle, $I C=$ inferior colliculus, PAG $=$ periaqueductal gray matter.

above may contribute to the linear and punctate midbrain lesions we have described.

In conclusion, we believe that small foci of high signal seen in the inferior and lateral midbrain represent enlarged perivascular spaces associated with penetrating branches of the collicular and accessory collicular arteries. Such lesions should not be mistaken for lacunar infarcts or nonspecific white matter changes when seen in this characteristic location.

\section{REFERENCES}

1. Braffman $B H$, Zimmerman RA, Trojanowski JQ, et al. Brain MR: pathologic correlation with gross and histopathology. 1. Lacunar infarction and Virchow-Robin spaces. AJNR 1988;9:621-628

2. Braffman BH, Zimmerman RA, Trojanowski JQ, et al. Brain MR: pathologic correlation with gross and histopathology. 2. Hyperintense white-matter foci in the elderly. AJNR 1988;9:629-636

3. Kirkpatrick JB, Hayman LA. White matter lesions in magnetic resonance imaging of clinically healthy brains of elderly subjects: possible pathologic basis. Radiology 1987; 162:509-511

4. Zimmerman RD, Fleming CA, Lee BCP, Saint-Louis LA, Deck MDF. Periventricular hyperintensity as seen by magnetic resonance: prevalence and significance. AJNR 1986;7:13-20

5. Sze G, DeArmond SJ, Brant-Zawadski M, et al. Foci of MRI signal (pseudo lesions) anterior to the frontal horns: histologic correlations of a normal finding. AJNR 1986;7:381-387

6. Salomon A, Yeates AE, Burger PC, Heinz ER. Subcortical arteriosclerotic encephalopathy: brain stem findings with MR imaging. Radiology 1987; $165: 625-629$

7. Jungreis CA, Kanal E, Hirsch WL, et al. Normal perivascular spaces mimicking lacunar infarction: MR imaging. Radiology 1988;169:101-104

8. Drayer BR. Imaging of the aging brain. I. Normal findings. Radiology 188;166:785-796

9. Heier LA, Bauer C, Schwartz L. Zimmerman RD, Deck MDF. Large Virchow-Robin spaces: MR-clinical correlation (abstr). AJNR 1988;9:1033.

10. Hachinski VC, Potter P, Merskey H. Leuko-araiosis. Arch Neurol 1987;44:21-24

11. Duvernoy HM. Human brainstem vesse/s. Berlin: Springer-Verlag, 1978:16-66 